\title{
AUTHOR INDEX Volume 18
}

Adegoke, B. O. A., Offiah, M. N., Okoye, E. C. and Akosile, C. O., Intra-Rater Reliabilities and Concurrent Validity of the Universal Goniometer and Tape Measure for Measuring Cervical Active Range of Motion

18 (2015) 1550005

Akosile, C. O., see Adegoke, B. O. A.

Alagha, B. and Padhiar, N., Partial Tear of Plantar Fascia in a Professional Dancer

Alavi, N. M., Madani, M., Taghizadeh, M. and Sharif, M. R., Vitamin D Supplementation in Female Nurses: The Effects on Serum 25-Hydroxyvitamin D, and Non-Specific Musculoskeletal Pain

Al-Shanti, N., see Okoro, T.

18 (2015) 1550005

18 (2015) 1572001

Amadio, P. C., see Filius, A.

An, K.-N., see Filius, A.

Andrew, J. G., see Okoro, T.

Annear, P. T., see Falconer, T. M.

Appleyard, R., see Dannaway, J.

Beck, T. W., see Wages, N. P.

Breidahl, W. H., see Falconer, T. M.

Breloff, S. P. and Chou, L.-S., A Multi-Segmented Approach to the Quantification of Trunk Movement During Gait

18 (2015) 1550008

18 (2015) 1550007

18 (2015) 1550019

18 (2015) 1550019

18 (2015) 1550007

18 (2015) 1550020

18 (2015) 1550015

18 (2015) 1550016

18 (2015) 1550020

18 (2015) 1550009

18 (2015) 1550006

18 (2015) 1550016

Carr, J. C., see Wages, N. P.

Chappell, I., Lee, P., McIff, T. E., Toby, E. B. and Fischer, K. J., A Method for Comparison of Radiocarpal Cartilage T2 Relaxation Time Maps and Contact Pressure Distributions in Normal and Injured Wrists

18 (2015) 1550012

18 (2015) 1550009

18 (2015) 1550015

Dabirrahmani, D., see Dannaway, J.

Damrow, D., Liu, X.-C. and Fehr, S., Musculoskeletal Injuries and Related Biomechanics for Youth Baseball Pitching

Dannaway, J., Dabirrahmani, D., Sonnabend, D., Martin, A. and Appleyard, R., An Investigation into the Frictional Properties Between Bone and Various Orthopedic Implant Surfaces: Implant Stability

18 (2015) 1530001

18 (2015) 1550015

Dao, T. T. and Tho, M.-C. H. B., Assessment of Parameter Uncertainty in Rigid Musculoskeletal Simulation Using a Probabilistic Approach

18 (2015) 1550013

18 (2015) 1572002

Dhanwate, A., see Menon, P. H.

18 (2015) 1550019

Dharan, A., see Filius, A.

18 (2015) 1550003

Edmondston, S., see Falconer, T. M.

18 (2015) 1550017 
Evis, Z., see Findikoglu, G.

18 (2015) 1550004

Falconer, T. M., Headford, J., Edmondston, S. and Yates, P. J., Validity of the Retrospective Application of Oxford Hip and Knee Scores

18 (2015) 1550003

Falconer, T. M., Tusak, L., Breidahl, W. H. and Annear, P. T., The LARS Augmented 4-Tunnel Hamstring "Hybrid" ACLR Graft Construction Allows Accelerated Rehabilitation Without Knee Laxity - Case Series of 111 Patients After 2 Years

18 (2015) 1550020

18 (2015) 1530001

Filius, A., Thoreson, A., Dharan, A., Mara, K., An, K.-N., Zhao, C. and Amadio, P. C., The Effect of Freezing on Biomechanical Properties of the Carpal Tunnel Subsynovial Connective Tissue

18 (2015) 1550019

Findikoglu, G. and Evis, Z., Microhardness Evaluation of Non Enzymatically Glycated Bovine Femur Cortical Bone

Fischer, K. J., see Chappell, I.

Gomes, J. L. E., see Muller, L.

Headford, J., see Falconer, T. M.

Homayouni, K., Naseri, M., Zaravar, F., Zaravar, L. and Karimian, H., Comparison of the Effect of Aquatic Physical Therapy and Conventional Physical Therapy in Patients with Lumbar Spinal Stenosis (A Randomized Controlled Trial)

Jain, P., see Sareen, A.

Jaramillo-Isaza, S., Mazeran, P.-E., El-Kirat, K. and Tho, M.-C. H. B., Heterogeneity of Time-Dependent Mechanical Properties of Human Cortical Bone at the Micro Scale

Karimian, H., see Homayouni, K.

Kehinde, E. O., see Khallaf, F. G.

Khallaf, F. G. and Kehinde, E. O., Healing of Long Bone Fractures in Patients with Concomitant Head or Spinal Cord Injuries

Kim, S.-S., see Moon, M.-S.

Kim, S.-S., see Moon, M.-S.

Koch, A. J., Machado, M. and Mayhew, J. L., Comparison between Bench Press and Leg Press for Changes in Serum Creatine Kinase Activity and Muscle Soreness

Kwon, K.-T., see Moon, M.-S.

Lai, D., see Senanayake, S. M. N. A.

Lee, P., see Chappell, I.

Lemmey, A., see Okoro, T.

Liu, X.-C., see Damrow, D.

Machado, M., see Koch, A. J.

Madani, M., see Alavi, N. M.

Maddison, P., see Okoro, T.

Manoj, M. K., see Menon, P. H.

Mara, K., see Filius, A.

Martin, A., see Dannaway, J.

Mayhew, J. L., see Koch, A. J.

Mazeran, P.-E., see Jaramillo-Isaza, S.

McIff, T. E., see Chappell, I.

Menon, P. H., Salunke, A. A., Murphy, D. P., Roy, S. P., Manoj, M. K., Nambi, G. I., Raval, P. and Dhanwate, A., A Missed Diagnosis of Asymptomatic Simultaneous Bilateral Fracture Neck Femur Due to Vitamin D Deficiency Treated as Severe Bilateral Osteoarthritis Knee: How to Prevent It?

18 (2015) 1550004

18 (2015) 1550012

18 (2015) 1550006

18 (2015) 1550003

18 (2015) 1550002

18 (2015) 1550010

18 (2015) 1550017

18 (2015) 1550002

18 (2015) 1550018

18 (2015) 1550018

18 (2015) 1550001

18 (2015) 1530002

18 (2015) 1550011

18 (2015) 1550001

18 (2015) 1550014

18 (2015) 1550012

18 (2015) 1550007

18 (2015) 1530001

18 (2015) 1550011

18 (2015) 1550008

18 (2015) 1550007

18 (2015) 1572002

18 (2015) 1550019

18 (2015) 1550015

18 (2015) 1550011

18 (2015) 1550017

18 (2015) 1550012

18 (2015) 1572002

18 (2015) 1550016

Miller, C. A., see Wages, N. P. 
Moon, M.-S., Kim, S.-S. and Sihn, J.-C., Tuberculous Kyphosis - Evolving Concepts in Prevention and Treatment

Moon, M.-S., Yoon, M.-K., Kwon, K.-T., Park, M.-S., Park, B.-K. and Kim, S.-S., Posterior Element Morphology and Degenerative Lumbar Spondylolisthesis

18 (2015) 1530002

18 (2015) 1550001

Muller, L., Caputo, G., Zago, M. K. and Gomes, J. L. E., Lateral Opening Wedge Distal Femoral V-Osteotomy: Biomechanics Comparison Between Angles of $60^{\circ}$ and $90^{\circ}$ in Sheep

Murphy, D. P., see Menon, P. H.

Nambi, G. I., see Menon, P. H.

Naseri, M., see Homayouni, K.

Offiah, M. N., see Adegoke, B. O. A.

Okoro, T., Stewart, C., Al-Shanti, N., Lemmey, A., Maddison, P. and Andrew, J. G., Objective Function, Lean Mass, and Associated Genetic Adaptations of the Operated Leg Following Total Hip Arthroplasty

Okoye, E. C., see Adegoke, B. O. A.

Padhiar, N., see Alagha, B.

Pagare, V., see Sareen, A.

Park, B.-K., see Moon, M.-S.

Park, M.-S., see Moon, M.-S.

Raval, P., see Menon, P. H.

Roy, S. P., see Menon, P. H.

Salunke, A. A., see Menon, P. H.

Sareen, A., Jain, P. and Pagare, V., Immediate Effect of Kinesiology Taping in Treating Undisplaced Rib Fracture Pain

Senanayake, S. M. N. A., Triloka, J. and Lai, D., Influence of Knee Flexion During Squat with and Without Load Using an Integrated Knee-Flexion Analysis System

Sharif, M. R., see Alavi, N. M.

Sihn, J.-C., see Moon, M.-S.

Sonnabend, D., see Dannaway, J.

Stewart, C., see Okoro, T.

Taghizadeh, M., see Alavi, N. M.

Tho, M.-C. H. B., see Dao, T. T.

Tho, M.-C. H. B., see Jaramillo-Isaza, S.

Thoreson, A., see Filius, A.

Toby, E. B., see Chappell, I.

Triloka, J., see Senanayake, S. M. N. A.

Tusak, L., see Falconer, T. M.

Wages, N. P., Beck, T. W., Ye, X., Carr, J. C. and Miller, C. A., Resting

Mechanomyographic Amplitude from the Vastus Lateralis Muscle in Different

Postural Resting Positions Following Aerobic Exercise

Yates, P. J., see Falconer, T. M.

Ye, X., see Wages, N. P.

Yoon, M.-K., see Moon, M.-S.

Zago, M. K., see Muller, L.

Zaravar, F., see Homayouni, K.

Zaravar, L., see Homayouni, K.

Zhao, C., see Filius, A.

18 (2015) 1550006

18 (2015) 1572002

18 (2015) 1572002

18 (2015) 1550002

18 (2015) 1550005

18 (2015) 1550007

18 (2015) 1550005

18 (2015) 1572001

18 (2015) 1550010

18 (2015) 1550001

18 (2015) 1550001

18 (2015) 1572002

18 (2015) 1572002

18 (2015) 1572002

18 (2015) 1550010

18 (2015) 1550014

18 (2015) 1550008

18 (2015) 1530002

18 (2015) 1550015

18 (2015) 1550007

18 (2015) 1550008

18 (2015) 1550013

18 (2015) 1550017

18 (2015) 1550019

18 (2015) 1550012

18 (2015) 1550014

18 (2015) 1550020

18 (2015) 1550016

18 (2015) 1550003

18 (2015) 1550016

18 (2015) 1550001

18 (2015) 1550006

18 (2015) 1550002

18 (2015) 1550002

18 (2015) 1550019 